Draft Version July 6, 2021

Preprint typeset using $\mathrm{IAT}_{\mathrm{E}} \mathrm{X}$ style emulateapj v. 5/2/11

\title{
ION COLLISIONAL TRANSPORT COEFFICIENTS IN THE SOLAR WIND AT 1 AU
}

\author{
Petr Hellinger \\ Astronomical Institute, CAS, Bocni II/1401, CZ-14100 Prague, Czech Republic \\ Draft version July 6, 2021
}

\begin{abstract}
Proton and alpha particle collisional transport coefficients (isotropization, relative deceleration frequencies and heating rates) at $1 \mathrm{AU}$ are quantified using the WIND/SWE data. In agreement with previous studies the ion-ion Coulomb collisions are generally important for slow solar wind streams and tend to reduce the temperature anisotropies, the differential streaming and the differences between proton and alpha particle temperatures. In slow solar wind streams the Coulomb collisions between protons and alpha particles are important for the overall proton energetics as well as for the relative deceleration between the two species. It is also shown that ion temperature anisotropies and differential streaming need to be generally taken into account for evaluation of the collisional transport coefficients.
\end{abstract}

\section{INTRODUCTION}

The solar wind plasma is weakly collisional and far from thermal equilibrium; the observed particle velocity distribution functions exhibit important temperature anisotropies and differential streaming between different populations (Marsch 2006, Hellinger et al. 2006). While the solar wind electrons are relatively strongly collisional, ions are essentially collisionless, except in slow solar wind streams (Neugebauer 1976, Marsch \& Goldstein 1983 Livi \& Marsch 1986). However, many ion properties in the solar wind are relatively well ordered by the ion collisionality measured through an estimated collisional age (Kasper et al. 2008). On the other hand, as many solar wind properties are correlated (cf., Hellinger \& Trávníček 2014 ) the role of collisions in the solar wind is not clear.

Transport in weakly collisional plasmas may strongly deviate from theoretical predictions obtained for collision-dominated plasmas (Spitzer \& Härm 1953: Braginskii 1965). Even for the relatively strongly collisional electrons such predictions (derived as a perturbation of thermal equilibrium) generally fail (cf., Landi et al. 2014). The collisional energy and momentum transport coefficients can be calculated by taking moments of the collisional operator assuming a particular form of particle distribution functions (Kogan 1961; Lehner 1967. Barakat \& Schunk 1981; Hernandez \& Marsch 1985). For drifting bi-Maxwellian velocity distribution functions these transport coefficients can be derived in in a closed form involving generalized double hypergeometric functions (Hellinger \& Trávníček 2009).

For modeling of the solar wind, simplified versions of the transport coefficients which neglect the temperature anisotropy and/or the differential streaming are often used (Hernandez et al. 1987, Echim et al. 2011; Maruca et al. 2013; Cranmer 2014; Tracy et al. 2015). Observed important ion temperature anisotropies and differential streaming make such approaches questionable (cf., Matteini et al. 2012). In this paper we quantify the collisional energy and momentum transport coefficients in the solar wind using WIND/SWE observations at 1 AU. This paper is organized as follows: section 2 gives an overview

petr.hellinger@asu.cas.cz of theoretical collisional transport coefficients for drifting bi-Maxwellian velocity distribution functions; in this section the collisional isotropization, relative deceleration and heating frequencies are defined. Section 3 presents an analysis of the WIND/SWE data for proton and alpha particles. The different collision frequencies are evaluated and compared with each other. The error due to neglecting the temperature anisotropy and/or the differential streaming is estimated. Section 4 summarizes and discusses the results.

\section{TRANSPORT COEFFICIENTS}

Here we assume a homogeneous plasma consisting of species with bi-Maxwellian velocity distribution functions $f_{\mathrm{s}}$ drifting along the magnetic field (here subscripts $\mathrm{s}$ and $\mathrm{t}$ denote different species; subscripts $\|$ and $\perp$ denote directions with respect to the ambient magnetic field):

$$
f_{\mathrm{s}}\left(v_{\|}, v_{\perp}\right)=\frac{n_{\mathrm{s}}}{(2 \pi)^{3 / 2} v_{\mathrm{s} \|} v_{\mathrm{s} \perp}^{2}} \mathrm{e}^{-\frac{v_{\perp}^{2}}{2 v_{\mathrm{s} \perp}^{2}}-\frac{\left(v_{\|}-v_{\mathrm{s}}\right)^{2}}{2 v_{\mathrm{s} \|}^{2}}}
$$

where $n_{\mathrm{s}}$ is the species number density, $v_{\mathrm{s} \|}=$ $\left(k_{B} T_{\mathrm{s} \|} / m_{\mathrm{s}}\right)^{1 / 2}$ and $v_{\mathrm{s} \perp}=\left(k_{B} T_{\mathrm{s} \perp} / m_{\mathrm{s}}\right)^{1 / 2}$ are the parallel and perpendicular thermal velocities corresponding to the parallel and perpendicular temperatures, $T_{\mathrm{s} \|}$ and $T_{\mathrm{s} \perp}$, respectively; $k_{B}$ is the Boltzmann constant, $m_{\mathrm{s}}$ is the mass, and $v_{\mathrm{s}}$ is the parallel drift velocity. For these distribution functions the collisional transport coefficients for the parallel and perpendicular temperatures may be given as (cf., Hellinger \& Trávníček 2009)

$$
\begin{aligned}
& \left(\frac{\mathrm{d} T_{\mathrm{s} \perp}}{\mathrm{d} t}\right)_{c}=-\nu_{T \mathrm{~s}}\left(T_{\mathrm{s} \perp}-T_{\mathrm{s} \|}\right)+T_{\mathrm{s} \perp} \sum_{\mathrm{t} \neq \mathrm{s}} \nu_{H \mathrm{~s} \perp}^{(\mathrm{t})} \\
& \left(\frac{\mathrm{d} T_{\mathrm{s} \|}}{\mathrm{d} t}\right)_{c}=2 \nu_{T \mathrm{~s}}\left(T_{\mathrm{s} \perp}-T_{\mathrm{s} \|}\right)+T_{\mathrm{s} \|} \sum_{\mathrm{t} \neq \mathrm{s}} \nu_{H \mathrm{~s} \|}^{(\mathrm{t})}
\end{aligned}
$$

where $\nu_{T \mathrm{~s}}$ is the (intraspecies) isotropization frequency (cf. Kogan 1961)

$$
\nu_{T \mathrm{~s}}=\frac{q_{\mathrm{s}}^{4} n_{\mathrm{s}} \ln \Lambda_{\mathrm{ss}}}{30 \pi^{3 / 2} \epsilon_{0}^{2} m_{\mathrm{s}}^{2} v_{\mathrm{s} \|}^{3}}{ }_{2} F_{1}\left(\begin{array}{c}
2,3 / 2 \\
7 / 2
\end{array} \mid 1-A_{\mathrm{s}}\right)
$$


and ${ }_{2} F_{1}$ is the standard (Gauss) hypergeometric function. Here $\epsilon_{0}$ denotes the electric permittivity, $q_{\mathrm{s}}$ is the charge; $A_{\mathrm{s}}=T_{\mathrm{s} \perp} / T_{\mathrm{s} \|}$ is the temperature anisotropy, and $\ln \Lambda_{\mathrm{ss}}$ is the Coulomb logarithm.

The energy transfer between the different species is quantified by the perpendicular and parallel heating rates $\nu_{H \mathrm{~s} \perp}^{(\mathrm{t})}$ and $\nu_{H \mathrm{~s} \|}^{(\mathrm{t})}$ which may be expressed analytically as

$$
\begin{aligned}
\nu_{H \mathrm{~s} \perp}^{(\mathrm{t})}= & \frac{\nu_{\mathrm{st}}}{A_{\mathrm{st}}}\left[\frac{m_{\mathrm{st}}}{m_{\mathrm{t}}}\left(\frac{T_{\mathrm{t} \perp}}{T_{\mathrm{s} \perp}}-1\right) F_{2 \frac{1}{2} \frac{5}{2}}^{(\mathrm{st})}+F_{2 \frac{1}{2} \frac{5}{2}}^{(\mathrm{st})}-F_{1 \frac{1}{2} \frac{5}{2}}^{(\mathrm{st})}\right. \\
\nu_{H \mathrm{~s} \|}^{(\mathrm{t})}= & \nu_{\mathrm{st}}\left[\frac{m_{\mathrm{st}}}{m_{\mathrm{t}}}\left(\frac{T_{\mathrm{t} \|}}{T_{\mathrm{s} \|}}-1\right) F_{1 \frac{1}{2} \frac{5}{2}}^{(\mathrm{st})}-2\left(F_{2 \frac{1}{2} \frac{5}{2}}^{(\mathrm{st})}-F_{1 \frac{1}{2} \frac{5}{2}}^{(\mathrm{st})}\right)\right. \\
& \left.+\frac{v_{\mathrm{st}}^{2}}{2 v_{\mathrm{st} \|}^{2}} F_{1 \frac{3}{2} \frac{5}{2}}^{(\mathrm{st})}\right] .
\end{aligned}
$$

Here

$$
v_{\mathrm{st} \|}=\sqrt{\frac{v_{\mathrm{s} \|}^{2}+v_{\mathrm{t} \|}^{2}}{2}} \text { and } v_{\mathrm{st} \perp}=\sqrt{\frac{v_{\mathrm{s} \perp}^{2}+v_{\mathrm{t} \perp}^{2}}{2}}
$$

are combined parallel and perpendicular thermal velocities, respectively, $v_{\mathrm{st}}=v_{\mathrm{s}}-v_{\mathrm{t}}$ is the relative velocity between the two species, $m_{\mathrm{st}}=m_{\mathrm{s}} m_{\mathrm{t}} /\left(m_{\mathrm{s}}+m_{\mathrm{t}}\right)$ is a combined mass

$$
A_{\mathrm{st}}=\frac{v_{\mathrm{st} \perp}^{2}}{v_{\mathrm{st} \|}^{2}}=\frac{m_{\mathrm{t}} T_{\mathrm{s} \perp}+m_{\mathrm{s}} T_{\mathrm{t} \perp}}{m_{\mathrm{t}} T_{\mathrm{s} \|}+m_{\mathrm{s}} T_{\mathrm{t} \|}}
$$

is a combined temperature anisotropies, and

$$
\nu_{\mathrm{st}}=\frac{q_{\mathrm{s}}^{2} q_{\mathrm{t}}^{2} n_{\mathrm{t}}}{12 \pi^{3 / 2} \epsilon_{0}^{2} m_{\mathrm{s}} m_{\mathrm{st}} v_{\mathrm{st} \|}^{3}} \ln \Lambda_{\mathrm{st}}
$$

is a collision frequency of species $\mathrm{s}$ on species $\mathrm{t}\left(\ln \Lambda_{\mathrm{st}}\right.$ being the corresponding Coulomb logarithm). Finally, $F_{a b c}^{(\text {st) }}$ are defined through generalized double hypergeometric or Kampé de Fériet functions (Exton 1976)

$$
F_{a b c}^{(\mathrm{st})}=\mathrm{e}^{-\frac{v_{\mathrm{st}}^{2}}{4 v_{\mathrm{st}}^{2} \|}} F_{1 \cdot 1}^{2 \cdot .}\left(\begin{array}{c}
a, b \\
c ; b
\end{array} \mid 1-A_{\mathrm{st}}, A_{\mathrm{st}} \frac{v_{\mathrm{st}}^{2}}{4 v_{\mathrm{st} \|}^{2}}\right) .
$$

These functions can be represented as double series

$$
F_{1 \cdot 1}^{2 . .}\left(\begin{array}{c}
a, b \\
c ; d
\end{array} \mid x, y\right)=\sum_{n, k=0}^{\infty} \frac{(a)_{n+k}(b)_{n+k}}{(c)_{n+k}(d)_{k}} \frac{x^{n}}{n !} \frac{y^{k}}{k !} .
$$

where $(a)_{n}$ denotes the Pochhammer symbol

$$
(a)_{n}=\frac{\Gamma(a+n)}{\Gamma(a)}=a(a+1) \ldots(a+n-1),
$$

$\Gamma$ being the gamma function. These series are absolutely convergent for any $y$ and for $|x|<1$. Outside this region an analytic continuation is needed. For the special case $b=d$ needed here, there exists a simple integral representation (Hellinger \& Trávníček 2009):

$$
\begin{aligned}
F_{1 \cdot 1}^{2 . .}\left(\begin{array}{c}
a, b \\
c ; b
\end{array} \mid x, y\right) & =\frac{\Gamma(c)}{\Gamma(a) \Gamma(c-a)} \\
& \times \int_{0}^{1} \frac{t^{a-1}(1-t)^{c-a-1}}{(1-t x)^{b}} \mathrm{e}^{\frac{t y}{1-t x}} \mathrm{~d} t .
\end{aligned}
$$

which may be used for numerical evaluation.

We also define the mean heating rate $\nu_{H \mathrm{~s}}^{(\mathrm{t})}$ as

$$
\nu_{H \mathrm{~s}}^{(\mathrm{t})}=\frac{1}{3} \frac{T_{\mathrm{s} \|}}{T_{\mathrm{s}}} \nu_{H \mathrm{~s} \|}^{(\mathrm{t})}+\frac{2}{3} \frac{T_{\mathrm{s} \perp}}{T_{\mathrm{s}}} \nu_{H \mathrm{~s} \perp}^{(\mathrm{t})}
$$

where $T_{\mathrm{s}}=\left(2 T_{\mathrm{s} \perp}+T_{\mathrm{s} \|}\right) / 3$ is the mean temperature of the species.

For the relative deceleration between species $\mathrm{s}$ and t through Coulomb collisions, one gets Hellinger \& Trávníček (2009)

$$
\left(\frac{\mathrm{d} v_{\mathrm{st}}}{\mathrm{d} t}\right)_{c}^{(\mathrm{st})}=-\nu_{V}^{(\mathrm{st})} v_{\mathrm{st}}
$$

where the deceleration frequency $\nu_{V}^{(\mathrm{st})}$ may be given as

$$
\nu_{V}^{\text {(st) }}=\frac{q_{\mathrm{s}}^{2} q_{\mathrm{t}}^{2} n_{\mathrm{st}}}{24 \pi^{3 / 2} \epsilon_{0}^{2} m_{\mathrm{st}}^{2} v_{\mathrm{st} \|}^{3}} \ln \Lambda_{\mathrm{st}} F_{1 \frac{3}{2} \frac{5}{2}}^{(\mathrm{st})}
$$

where $n_{\mathrm{st}}=\left(n_{\mathrm{s}} m_{\mathrm{s}}+n_{\mathrm{t}} m_{\mathrm{t}}\right) /\left(m_{\mathrm{s}}+m_{\mathrm{t}}\right)$ is a combined number density.

\section{COLLISIONAL TRANSPORT COEFFICIENTS}

Here we use fitted data from the two Faraday Cup instruments in the Solar Wind Experiment (SWE) on the Wind spacecraft. WIND is a rotating spacecraft with a spin-axis perpendicular to the ecliptic plane and a period of three seconds. A Faraday Cup is an energy/charge instrument with a large, conical field of view which measures the current produced by particles within a given energy window. Proton and alpha particle properties, number densities and parallel and perpendicular temperatures are obtained using a non-linear least-squares fitting of data to a theoretical model assuming bi-Maxwellian proton and alpha particle distribution functions and the magnetic field direction obtained from three-second measurements provided by the Magnetic Field Investigation on the Wind spacecraft. In this paper we use a large statistical data set (about 4 millions data points) from 1995 to 2012 (Kasper et al. 2008: Maruca et al. 2012). We use only the data when the Wind spacecraft was situated in the solar wind at about $1 \mathrm{AU}$ (portions of time when it was inside the magnetosphere before 2004 were removed from the data set).

For the fitted proton and alpha particle parameters we calculate the different transport coefficients (given in section 20 approximating the ion-ion Coulomb logarithm by (cf., Tracy et al. 2015)

$$
\ln \Lambda_{\mathrm{st}}=29.9-\ln \left[\frac{q_{\mathrm{s}} q_{\mathrm{t}}\left(m_{\mathrm{s}}+m_{\mathrm{t}}\right)}{e^{3}\left(m_{\mathrm{s}} \tilde{T}_{\mathrm{t}}+m_{\mathrm{t}} \tilde{T}_{\mathrm{s}}\right)}\left(\frac{n_{\mathrm{s}} q_{\mathrm{s}}^{2}}{\tilde{T}_{\mathrm{s}}}+\frac{n_{\mathrm{t}} q_{\mathrm{t}}^{2}}{\tilde{T}_{\mathrm{t}}}\right)^{1 / 2}\right]
$$


where the temperature anisotropies and differential streaming are neglected. In this expression $\tilde{T}_{\mathrm{S}}$ stands for the temperature of species $\mathrm{s}$ in electrovolts and $e$ is the proton charge. For numerical evaluation of the generalized double hypergeometric functions we use the integral representation, Eq. (13).

We start with the proton-alpha particle deceleration frequency $\nu_{V}^{(\alpha \mathrm{p})}$ (henceforth we drop the superscript) with respect to the characteristic transit/expansion time $t_{e}=R / v_{s w}$ where $R$ is radial distance from the sun (being $1 \mathrm{AU}$ here) and $v_{s w}$ is the solar wind velocity. The product of a collisional transport coefficient such as $\nu_{V}$ and the expansion time $t_{e}$ may be used as a proxy for the collisional age (cf., Kasper et al. 2008), defined as an integral value over a relevant time interval $\left(t_{0}, t\right)$ of a given collisional frequency $\nu A_{c}=\int_{t_{0}}^{t} \nu\left(t^{\prime}\right) \mathrm{d} t^{\prime}$ (cf., Salem et al. 2003 Chhiber et al. 2016); here we prefer to interpret $\nu_{V} t_{e}$ (and other such products) as a way to compare the two local characteristic times.

The left panel of Figure 1 shows the distribution of data in the space $\left(v_{s w}, \nu_{V} t_{e}\right)$. This distribution (and all the following ones) was obtained by calculating number of data points in each bin and dividing it by the bin size (cf., Maruca et al. 2012); the results are then globally renormalized to have the maximum value of the distribution equal to 1 . We recover the well known result that slower streams are typically more collisional. It is in-
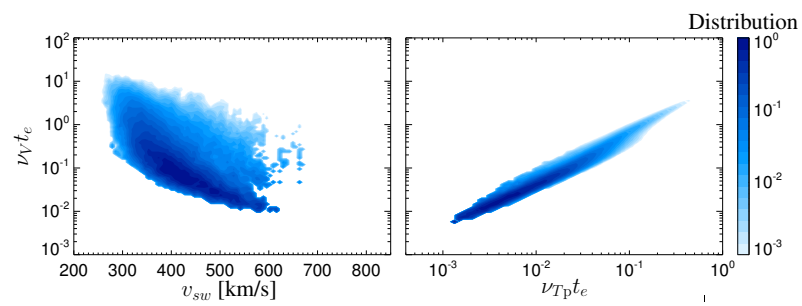

Figure 1. Proton-alpha particle deceleration frequency: Color scale plots of the relative frequency of $\left(v_{s w}, \nu_{V} t_{e}\right)$ (left panel) and of $\left(\nu_{T \mathrm{p}} t_{e}, \nu_{V} t_{e}\right)$ (right panel).

teresting to compare the relative deceleration frequency $\nu_{V}$ and the (proton-proton) isotropization frequency $\nu_{T \mathrm{p}}$ The right panel of Figure 1 shows the distribution of data in the space $\left(\nu_{T \mathrm{p}} t_{e}, \nu_{V} t_{e}\right)$ (cf., Hellinger \& Trávníček 2014). The two collisional frequencies are almost proportional to each other as expected. Note that the deceleration frequency $\nu_{V}$ is noticeably larger than the proton isotropization one $\nu_{T \mathrm{p}}$ (cf., Matteini et al. 2012).

The relative proton-alpha particle velocity in the solar wind tends to decrease with the radial distance at a rate similar to that of the Alfvén velocity $v_{A}$ (Marsch et al. 1982a: Verscharen et al. 2015). It it therefore interesting to compare the collisional rate $\left(\mathrm{d} v_{\alpha \mathrm{p}} / \mathrm{d} t\right)_{c}^{(\alpha \mathrm{p})}$ and the decrease rate $\mathrm{d} v_{A} / \mathrm{d} t=v_{s w} \mathrm{~d} v_{A} / \mathrm{d} R$; let us investigate the ratio of the two rates:

$$
\eta=\left|\frac{\nu_{V} v_{\alpha \mathrm{p}}}{v_{s w} \mathrm{~d} v_{A} / \mathrm{d} R}\right| .
$$

For the evaluation of $\eta$ we assume that the ion number densities decreases as $R^{-2}$ and that the magnetic field follows the Parker spiral with the angle $45^{\circ}$ at $1 \mathrm{AU}$. The obtained results are shown in Figure 2 where the left panel shows the distribution of data in the space $\left(v_{s w}, \eta\right)$ whereas the right panels the distribution in ( $\left.\left|v_{\alpha \mathrm{p}}\right| / v_{A}, \eta\right)$. Figure 2 indicate that Coulomb collisions between protons and alpha parties may be sufficient to decelerate the two species with respect to each other at a rate comparable to the decrease rate of $v_{A}$ in some slow solar wind streams.

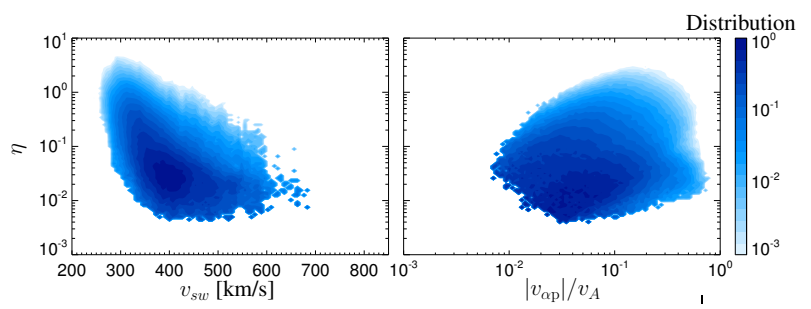

Figure 2. Proton-alpha deceleration with respect to the Alfvén velocity $v_{A}$ : Color scale plots of the relative frequency of $\left(v_{s w}, \eta\right)$ (left panel) and of $\left(\left|v_{\alpha \mathrm{p}}\right| / v_{A}, \eta\right)$ (right panel).

For the alpha particle isotropization frequency $\nu_{T \alpha}$ one expects much smaller values than for the proton one owing to the smaller alpha particle abundance (and typically larger temperatures). The observations, indeed, show that $\nu_{T \alpha}$ is about an order of magnitude smaller than (and almost proportional to) $\nu_{T \mathrm{p}}$.

Let's now look at the heating rates due to the Coulomb collisions between protons and alpha particles. Figure 3 shows the data distribution in $\left(v_{s w}, \nu_{H \mathrm{p} \|}^{(\alpha)} t_{e}\right)$ (top left panel), $\left(\nu_{T \mathrm{p}} t_{e}, \nu_{H \mathrm{p} \|}^{(\alpha)} t_{e}\right)$ (top right panel), $\left(v_{s w}, \nu_{H \mathrm{p} \perp}^{(\alpha)} t_{e}\right)$ (middle left panel), $\left(\nu_{T \mathrm{p}} t_{e}, \nu_{H \mathrm{p} \perp}^{(\alpha)} t_{e}\right)$ (middle right panel), $\left(v_{s w}, \nu_{H \mathrm{p}}^{(\alpha)} t_{e}\right)$ (bottom left panel), and in $\left(\nu_{T \mathrm{p}} t_{e}, \nu_{H \mathrm{p}}^{(\alpha)} t_{e}\right)$ (bottom right panel). Figure 3 indicates that protons (in slower, more collisional streams) are typically heated (in total) through collisions with alpha particles. This is quite natural as alpha particles are usually hotter than protons and collisions tend to remove this difference. Protons are sometimes cooled in either parallel or perpendicular directions; this happens typically in the cases of parallel or perpendicular proton temperature anisotropy. Proton collisions with alpha particles also tend to reduce the proton temperature anisotropy.

Figure 4 shows the data distribution in $\left(v_{s w}, \nu_{H \alpha \|}^{(\mathrm{p})} t_{e}\right)$ (top left panel), $\left(\nu_{T \mathrm{p}} t_{e}, \nu_{H \alpha \|}^{(\mathrm{p})} t_{e}\right)$ (top right panel), $\left(v_{s w}\right.$, $\left.\nu_{H \alpha \perp}^{(\mathrm{p})} t_{e}\right)$ (middle left panel), $\left(\nu_{T \mathrm{p}} t_{e}, \nu_{H \alpha \perp}^{(\mathrm{p})} t_{e}\right)$ (middle right panel), $\left(v_{s w}, \nu_{H \alpha}^{(\mathrm{p})} t_{e}\right)$ (bottom left panel), and in $\left(\nu_{T \mathrm{p}} t_{e}, \nu_{H \alpha}^{(\mathrm{p})} t_{e}\right)$ (bottom right panel). Figure 4 indicates that alpha particles (in slower, more collisional streams) are often cooled through collisions with protons; the energy stored in the proton-alpha particle differential velocity (as well as a part of the alpha particle thermal energy) goes most probably to protons. The cases when alpha particles are heated correspond typically to the cases when protons and alpha particles have comparable temperatures. In these cases the differential streaming energies is split to protons and alpha particles. Alpha particles are sometimes heated in either parallel or perpendicular directions; this happens typically in the cases of perpendicular or parallel alpha particle temperature anisotropy. Alpha particle collisions with protons also 


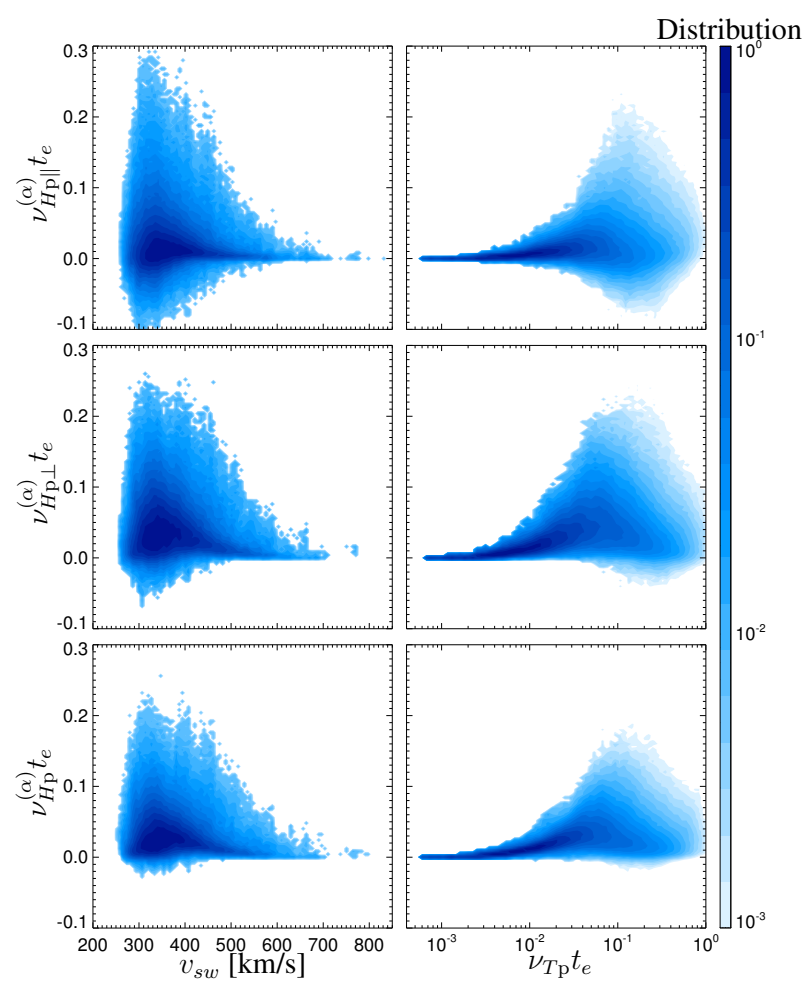

Figure 3. Proton heating rates through collisions with alpha particles: Color scale plots of the data distributions in $\left(v_{s w}, \nu_{H \mathrm{p} \|}^{(\alpha)} t_{e}\right)$ (top left panel), $\left(\nu_{T \mathrm{p}} t_{e}, \nu_{H \mathrm{p} \|}^{(\alpha)} t_{e}\right)$ (top right panel), $\left(v_{s w}, \nu_{H \mathrm{p} \perp}^{(\alpha)} t_{e}\right)$ (middle left panel), $\left(\nu_{T \mathrm{p}} t_{e}, \nu_{H \mathrm{p} \perp}^{(\alpha)} t_{e}\right)$ (middle right panel), $\left(v_{s w}\right.$, $\left.\nu_{H \mathrm{p}}^{(\alpha)} t_{e}\right)$ (bottom left panel), and in $\left(\nu_{T \mathrm{p}} t_{e}, \nu_{H \mathrm{p}}^{(\alpha)} t_{e}\right)$ (bottom right panel).

tend to reduce the alpha particle temperature anisotropy.

The expressions for the different collisional transport coefficients are rather complex. It is therefore interesting to test whether it is necessary to take into account the temperature anisotropy or the differential streaming. We estimated the importance of these parameters assuming isotropic populations (with the mean temperature $\left.T_{\mathrm{s}}=\left(T_{\mathrm{s} \|}+2 T_{\mathrm{s} \perp}\right) / 3\right)$ and/or neglecting the differential ion streaming (i.e., setting $v_{\mathrm{p} \alpha}=0$ ) for the present data set. The relative error in the isotropization frequency $\nu_{T}$ is small ( $\lesssim 10 \%$ ) when ignoring the temperature anisotropy for protons and alpha particles. However, the relative error in the relative deceleration frequency $\nu_{V}$ can be quite large $(\lesssim 40 \%)$ when ignoring the temperature anisotropy and/or the differential streaming. In the case of the (total) collisional heating rates $\nu_{H}$ the difference between the full (drifting \& anisotropic) version and that which neglects the temperature anisotropy and/or the differential streaming, $\left|\Delta \nu_{H}\right|$ could be of the order of $0.1 / t_{e}$ for protons and $1 / t_{e}$ for alpha particles (the parallel and perpendicular heating rates have typically somewhat larger errors). These values are comparable to the maximum (absolute) values of the heating rates (see Figures 3 and 4 ).

\section{DISCUSSION}

We quantified proton and alpha particles collisional transport coefficients at 1 AU using the WIND/SWE data. In agreement with previous studies our results

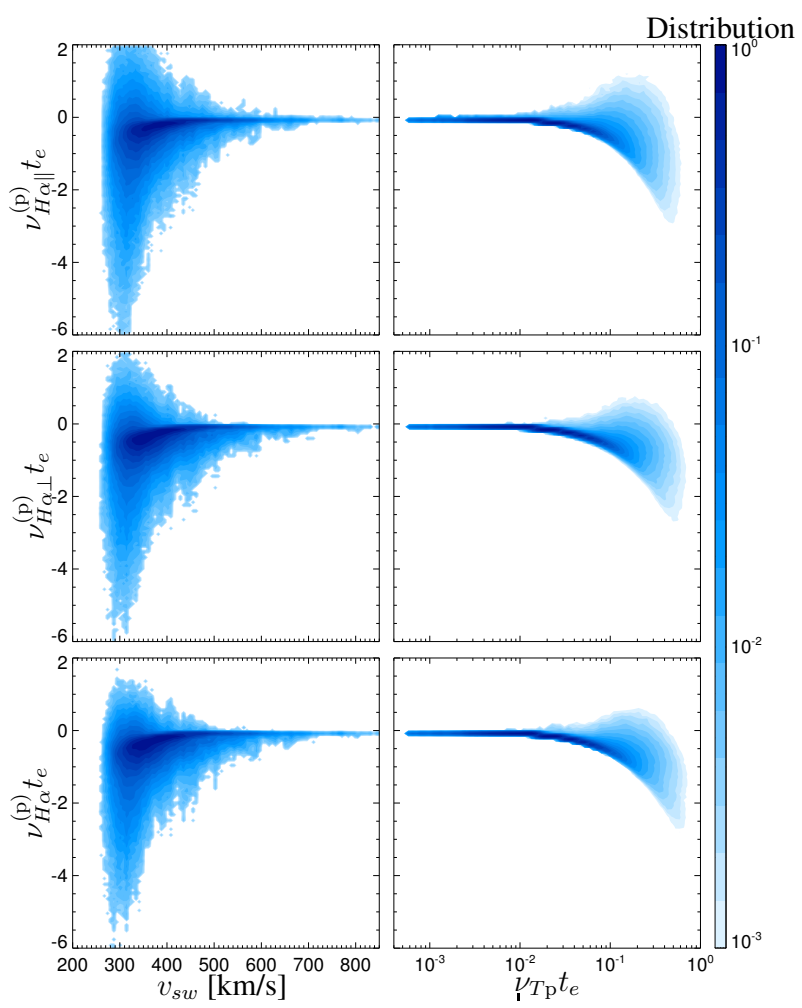

Figure 4. Alpha particle heating rates through collisions with protons: Color scale plots of the data distributions $\left(v_{s w}, \nu_{H \alpha \|}^{(\mathrm{p})} t_{e}\right)$ (top left panel), $\left(\nu_{T \mathrm{p}} t_{e}, \nu_{H \alpha \|}^{(\mathrm{p})} t_{e}\right)$ (top right panel), $\left(v_{s w}, \nu_{H \alpha \perp}^{(\mathrm{p})} t_{e}\right)$ (middle left panel), $\left(\nu_{T \mathrm{p}} t_{e}, \nu_{H \alpha \perp}^{(\mathrm{p})} t_{e}\right)$ (middle right panel), $\left(v_{s w}\right.$, $\left.\nu_{H \alpha}^{(\mathrm{p})} t_{e}\right)$ (bottom left panel), and in $\left(\nu_{T \mathrm{p}} t_{e}, \nu_{H \alpha}^{(\mathrm{p})} t_{e}\right)$ (bottom right panel).

show that ion-ion Coulomb collisions are generally important for slow solar wind streams; they tend to reduce the differences between the ion mean velocities and temperatures, as well as the ion temperature anisotropies. The different collisional frequencies have typically disparate values leading to different collisional ages. The two isotropization frequencies and the relative deceleration frequency are nearly proportional each to other so that one expects that the corresponding collisional times will be also proportional each to other. On the other hand, the heating rates are not simply related to these frequencies, and, moreover, these heating rates have positive and negative values.

The observations indicate that the relative protonalpha particle collisional deceleration is more efficient than the proton isotropization (and the alpha-particle isotropization) but, on the other hand, proton-alpha particle collisions also tend to reduce both the proton and alpha particle temperature anisotropies (in a relatively small number of cases the proton-alpha particle collisions enhance the ion temperature anisotropy). In some slow solar wind streams the Coulomb collisions are sufficiently strong to reduce the differential velocity between proton and alpha particles at a pace that is comparable to that of the decrease rate of the Alfvén velocity.

Protons are typically heated through collisions with alpha particles whereas alpha particles are very often cooled; this is a consequence of the temperature differ- 
ence between the two species, alpha particles are typically hotter than protons. When protons and alpha particles have comparable temperatures both the species are typically heated at the expense of the relative protonalpha particle velocity. Our results indicate that the proton heating through collisions with alpha particles in slow solar wind streams reaches values $\left(\mathrm{d} T_{\mathrm{p}} / \mathrm{d} t\right)_{c}^{(\alpha)} \sim$ $0.2 T_{\mathrm{p}} / t_{e}$ at $1 \mathrm{AU}$. This value is an important fraction of the needed average proton heating rate estimated from the Helios observations for an average slow solar wind $\mathrm{d} T_{\mathrm{p}} / \mathrm{d} t-\left(T_{\mathrm{p}} / \mathrm{d} t\right)_{\mathrm{CGL}} \sim 0.45 T_{\mathrm{p}} / t_{e}$ (cf., Hellinger et al. 2013). In the fast solar wind the needed proton heating rate has similar values $\sim 0.32 T_{\mathrm{p}} / t_{e}$ but the collisional proton heating rates are much smaller. Coulomb collisions with alpha particles are not energetically important for protons in the fast solar wind at $1 \mathrm{AU}$.

The collisional interaction of alpha particles with protons may be important in slow solar wind streams. Similarly, Tracy et al. (2015) show that Coulomb collision with protons may be also important for other minor ions. It is possible that the (summed effect of the) interaction between protons and other minor ions may have a nonnegligible heating effect on protons; the abundances of the other minor ions are much lower than the abundance of alpha particles but together they have about $1 \%$ and they are about mass proportionally hotter than alpha particles.

Our results show that the different parallel and perpendicular temperatures and differential velocities between ion species need to be taken into account for the collisional transport coefficients especially in slow/collisional solar wind streams. For the given data set of the WIND/SWE observations at 1 AU neglecting the ion temperature anisotropy and/or the differential streaming leads to relatively large errors. Consequently, it is important to use the general, anisotropic and drifting approximation for the collisional transport coefficients, particularly for modeling of slow solar wind streams where the induced error cumulates. The present results have (at best) theoretical uncertainties of the order of $1 / \ln \Lambda_{\text {st }}$. Equation (17) gives the uncertainties 4-5\% for the ionion interaction. Equation (17) is simplified, the temperature anisotropy and the differential streaming are neglected. It would be interesting to extend Eq. (17) to include these effect, but, on the other hand, the Coulomb logarithm is only weakly/logarithmically dependent on the plasma parameters, so that we do not expect significant changes. On the other hand, our model assumes biMaxwellian particle velocity distribution functions drifting with respect each other along the ambient magnetic field. If the ion distribution functions in the solar wind strongly depart from this model, these collisional transport coefficients are likely not applicable and further work is needed. For instance, the solar wind protons often consist of two populations (Marsch 2006); such a velocity distribution function can be to some extent modelled as a superposition of two drifting bi-Maxwellian velocity distribution functions and the present model can be directly applied.

PH acknowledges grant 15-10057S of the Grant Agency of the Czech Republic and the project RVO:67985815. The research leading to these results has received funding from the European Commission's 7th Framework Programme under the grant agreement \#284515 (projectshock.eu). Wind data were obtained from the NSSDC website http://nssdc.gsfc.nasa.gov.

\section{REFERENCES}

Barakat, A. R., \& Schunk, R. W. 1981, J. Phys. D: Appl. Phys., 14,421

Braginskii, S. I. 1965, in Rev. Plasma Phys., ed. M. Leontovich, Vol. 1 (New York: Consultants Bureau), 205-311

Chhiber, R., Usmanov, A. V., Matthaeus, W. H., \& Goldstein, M. L. 2016, ApJ, 821, 34

Cranmer, S. R. 2014, ApJS, 213, 16

Echim, M. M., Lemaire, J., \& Lie-Svendsen, Ø. 2011, Surv. Geophys., 32, 1

Exton, H. 1976 (New York: Halsted Press)

Hellinger, P., Trávníček, P., Kasper, J. C., \& Lazarus, A. J. 2006, Geophys. Res. Lett., 33, L09101

Hellinger, P., \& Trávníček, P. M. 2009, Phys. Plasmas, 16, 054501

—. 2014, ApJL, 784, L15

Hellinger, P., Trávníček, P. M., Štverák, Š., Matteini, L., \& Velli, M. 2013, J. Geophys. Res., 118, 1351

Hernandez, R., Livi, S., \& Marsch, E. 1987, J. Geophys. Res., 92, 7723

Hernandez, R., \& Marsch, E. 1985, J. Geophys. Res., 90, 11062

Kasper, J. C., Lazarus, A. J., \& Gary, S. P. 2008, Phys. Rev. Lett., 101, 261103

Kogan, V. I. 1961, in Plasma Physics and the Problem of Controlled Thermonuclear Reactions, ed. M. A. Leontovich, Vol. 1 (New York: Pergamon Press), 153-161

Landi, S., Matteini, L., \& Pantellini, F. 2014, ApJL, 790, L12

Lehner, G. 1967, Zeitschrift fur Physik, 206, 284

Livi, S., \& Marsch, E. 1986, Ann. Geophys., 4, 333

Marsch. E. 2006. LRSP. 3.

http://www.livingreviews.org/lrsp-2006-1

Marsch, $\bar{E}$., \& Goldstein, $\bar{H}$. 1983, J. Geophys. Res., 88, 9933

Marsch, E., Muhlhauser, K. H., Rosenbauer, H., Schwenn, R., \& Neubauer, F. M. 1982a, J. Geophys. Res., 87, 35

Marsch, E., Muhlhauser, K. H., Schwenn, R., Rosenbauer, H. Pilipp, W., \& Neubauer, F. M. 1982b, J. Geophys. Res., 87, 52

Maruca, B. A., Bale, S. D., Sorriso-Valvo, L., Kasper, J. C., \& Stevens, M. L. 2013, Phys. Rev. Lett., 241101

Maruca, B. A., Kasper, J. C., \& Gary, S. P. 2012, ApJ, 748, 137

Matteini, L., Hellinger, P., Landi, S., Trávníček, P. M., \& Velli, M. 2012, Space Sci. Rev., 172, 373

Neugebauer, M. 1976, J. Geophys. Res., 81, 78

Salem, C., Hubert, D., Lacombe, C., Bale, S. D., Mangeney, A., Larson, D. E., \& Lin, R. P. 2003, ApJ, 585, 1147

Spitzer, L., \& Härm, R. 1953, Phys. Rep., 89, 977

Tracy, P. J., Kasper, J. C., Zurbuchen, T. H., Raines, J. M., Shearer, P., \& Gilbert, J. 2015, ApJ, 812, 170

Verscharen, D., Chandran, B. D. G., Bourouaine, S., \& Hollweg, J. V. 2015, ApJ, 806, 157 